PREPARED FOR THE U.S. DEPARTMENT OF ENERGY, UNDER CONTRACT DE-AC02-76CH03073

PPPL-3845

PPPL-3845

UC-70

L-H Mode Transitions in the National Spherical Torus Experiment

by

S.M. Kaye, C.E. Bush, E. Fredrickson, B. LeBlanc,

R. Maingi, and S.A. Sabbagh

July 2003

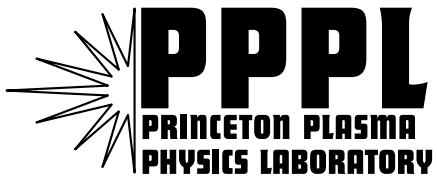

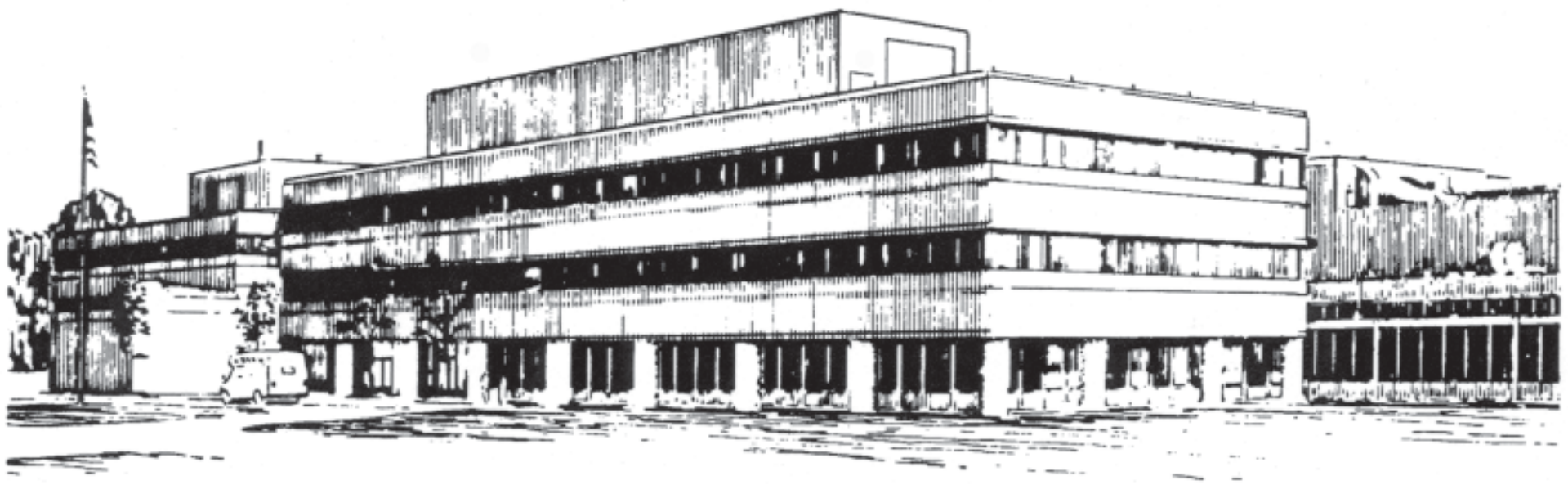

PRINCETON PLASMA PHYSICS LABORATORY PRINCETON UNIVERSITY, PRINCETON, NEW JERSEY 


\section{PPPL Reports Disclaimer}

This report was prepared as an account of work sponsored by an agency of the United States Government. Neither the United States Government nor any agency thereof, nor any of their employees, makes any warranty, express or implied, or assumes any legal liability or responsibility for the accuracy, completeness, or usefulness of any information, apparatus, product, or process disclosed, or represents that its use would not infringe privately owned rights. Reference herein to any specific commercial product, process, or service by trade name, trademark, manufacturer, or otherwise, does not necessarily constitute or imply its endorsement, recommendation, or favoring by the United States Government or any agency thereof. The views and opinions of authors expressed herein do not necessarily state or reflect those of the United States Government or any agency thereof.

\section{Availability}

This report is posted on the U.S. Department of Energy's Princeton Plasma Physics Laboratory Publications and Reports web site in Fiscal Year 2003. The home page for PPPL Reports and Publications is: http://www.pppl.gov/pub_report/

DOE and DOE Contractors can obtain copies of this report from:

U.S. Department of Energy

Office of Scientific and Technical Information

DOE Technical Information Services (DTIS)

P.O. Box 62

Oak Ridge, TN 37831

Telephone: (865) 576-8401

Fax: (865) 576-5728

Email: reports@adonis.osti.gov

This report is available to the general public from:

National Technical Information Service

U.S. Department of Commerce

5285 Port Royal Road

Springfield, VA 22161

Telephone: $1-800-553-6847$ or

(703) $605-6000$

Fax: (703) 321-8547

Internet: http://www.ntis.gov/ordering.htm 


\title{
L-H Mode Transitions in the National Spherical Torus Experiment (NSTX)
}

\author{
S.M. Kaye ${ }^{1}$, C.E. Bush ${ }^{2}$, E. Fredrickson ${ }^{1}$, B. LeBlanc ${ }^{1}$, R. Maingi ${ }^{2}$, S.A. Sabbagh ${ }^{3}$
}

\begin{abstract}
Edge data from plasmas in the National Spherical Torus Experiment (NSTX) [S. Kaye et al., Fusion Technology, 36, 16 (1999)] have been compared to theories of transport suppression that have been used to develop a physics framework for Low confinement (L)- to High confinement (H)-mode transitions. The NSTX data were obtained in low aspect ratio $(\mathrm{R} / \mathrm{a} \simeq 1.3)$ discharges taken from a variety of discharge phases, including L-modes, L-H transitions and H-modes with and without Edge Localized Modes (ELMs). The comparisons show that the group of points taken just before the L-H mode transition are well mixed with the purely L-mode group to within the measurement uncertainties, indicating that changes in these parameters leading up to the transition are subtle. One of the theory parameters, $\alpha_{M H D}=-R q^{2} d \beta_{t} / d r$, does show a clear threshold ( $\alpha_{M H D}=1$ to 2 ) between the H-mode grouping of points and those remaining in the L-mode or taken just prior to the transition. Additionally, there is no evidence for an edge temperature threshold necessary for transitioning into the H-mode. NSTX data indicate further a possible connection between L-H transitions and non-ambipolar beam ion losses.
\end{abstract}

\footnotetext{
${ }^{1}$ Princeton Plasma Physics Laboratory, Princeton University, Princeton, N.J. 08543

${ }^{2}$ Oak Ridge National Laboratory, Oak Ridge, Tenn.

${ }^{3}$ Dept. of Applied Physics and Applied Mathematics, Columbia Univ, NYC, NY, 10027
} 


\section{INTRODUCTION}

The discovery of the High-confinement $\left(\mathrm{H}^{-}\right)$mode $^{1}$ nearly two decades ago, as well as development of the "supershot"2 operating scenarios, have indicated the critical role of the plasma edge in optimizing confinement throughout the entire plasma. In particular, the H-mode offers an operating scenario that can combine high energy confinement, greater plasma stability to magnetohydrodynamic (MHD) modes and steady-state operation. It is, therefore, important to understand those discharge conditions and parameters that could lead to the transition from the Low confinement (L-) mode to the H-mode state. Since the H-mode transport barrier develops near the plasma edge, it is natural to study plasma edge parameters in this context.

There has been much work to develop theoretical models of transport suppression that both distinguish between the two confinement states, and, more importantly, that can lead to a predictive means of identifying conditions and parameter changes just prior to the transition between the two. The latter will help to identify the underlying physics mechanisms as well as facilitate device operation when $\mathrm{H}$-mode states either are, or are not, desired. Recent theory has focused on drift-resistive ballooning modes, ${ }^{3,4}$ peeling modes, ${ }^{5}$ the driftAlfvén instability ${ }^{6}$ and, most recently, on shear flow and field generation by finite- $\beta_{t}$ drift waves. $^{7}$

The theories have enabled experimentalists to characterize the measured edge parameters by the important variables as predicted by the theory in an effort to identify the underlying change of transport processes. For instance, results from Alcator C-Mod indicated a threshold edge $T_{e}$ for an L-H transition of approximately $120 \mathrm{eV}$ at the $95 \%$ flux surface, and clearly showed a distinction, within the framework of the drift-resistive ballooning mode theory, between discharges in well developed L- or H-mode plasmas. ${ }^{8}$ A simple temperature threshold was not observed in DIII-D, ${ }^{9,10}$ which, nevertheless, also showed a distinction between L- and H-mode phases for all three theories, but the results did not 
identify parameter changes as a precursor to an H-mode transition. ${ }^{9}$ Furthermore, the Hmode edge parameters for pellet-fueled discharges in DIII-D fell significantly below those predicted by the theories. ${ }^{10}$ On the other hand, data from COMPASS-D appears to show some predictive capability of the drift-Alfven theory in that discharge characteristics near the time of the L-H transition clustered at or above the predicted transition threshold. ${ }^{11}$ In the recent study of finite- $\beta_{t}$ drift wave-induced turbulence suppression, DIII-D edge temperatures showed very good agreement with the predicted threshold. ${ }^{7}$ The authors also indicated that this theory is successful in reconciling differences in the threshold dependence on $\nabla \mathrm{B}$ drift direction and pellet fueling.

Studies of possible temperature thresholds for L-H transitions have been carried out in other devices, ASDEX-U, ${ }^{12} \mathrm{JET}^{13}$ and JT-60U,,${ }^{14}$ although these studies did not attempt to relate the measured edge parameters to the predictions of the transition theories mentioned above.

In this paper, for the first time, data from $\mathrm{L}$ - and $\mathrm{H}$-modes in a high power spherical torus (i.e., low aspect ratio tokamak) will be studied in the framework of the theories discussed above. The data were obtained from experiments on the National Spherical Torus Experiment (NSTX), which has the following operating parameters: major/minor radius, $\mathrm{R} / \mathrm{a}=0.85 / 0.68 \mathrm{~m} \simeq 1.3$; plasma current, $I_{p} \leq 1.4 \mathrm{MA}$; toroidal magnetic field, $B_{T} \leq 0.6$ T; elongation, $\kappa \leq 2.2$; triangularity, $\delta \leq 0.8$; neutral beam heating power, $P_{N B} \leq 5 \mathrm{MW}$ ( $\leq 80 \mathrm{keV} D^{0}$ neutral beams); High Harmonic Fast Wave heating power, $P_{H H F W} \leq 6$ MW, and $D^{+}$plasmas. A complete description of the design and physics mission of the experiment can be found in Kaye et al. ${ }^{15}$ 


\section{EXPERIMENTAL RESULTS}

\section{A. H-mode Plasmas}

Attaining the H-mode in NSTX has been crucial to achieving a high- $\beta_{t}$, high noninductive current fraction discharge. As will be seen, H-mode operation in NSTX leads to broad density profiles, which, in turn, lead to broad pressure profiles and high- $q_{0}$ necessary for stable high- $\beta_{t}$ operation. High- $\beta_{t}$ operation, coupled with the high energy confinement times associated with H-modes (up to 2.5 times ITER97L), ${ }^{16}$ leads to relatively high bootstrap fractions. To date, $\left\langle\beta_{t}\right\rangle$ values up to $36 \%$ have been achieved, where $\beta_{t} \propto<p>/ B_{0}^{2}$, where $B_{0}$ is the vacuum toroidal magnetic field at the geometric axis. Corresponding values of $\beta_{N}$ (=a $\beta_{t} B_{0} / I_{p}$ ) have reached 6 to 6.5 in H-mode plasmas. ${ }^{17}$ Irrespective of the definition of $\beta_{t}$ (i.e., with respect to the toroidal or total magnetic field),

these high $\beta_{t}$ plasmas are above the ideal, no-wall limit. ${ }^{18}$ Additionally, H-mode discharges have achieved steady-state conditions for over $500 \mathrm{msec}\left(\geq 5 \tau_{E}\right)$, and bootstrap fractions of approximately $50 \%$.

H-mode plasmas were created in NSTX with both neutral beam (NB) and radio frequencey (rf) heating. In order to obtain an L-H transition with neutral beam heating, it was necessary to operate with plasmas in a lower single null or double null divertor configuration with a 1 to $6 \mathrm{~cm}$ inner wall gap, $I_{p}$ from 0.6 to $1.3 \mathrm{MA}, B_{T}$ from 0.3 to $0.6 \mathrm{~T}$ and $P_{N B}>0.36 \mathrm{MW}$. Densities at the transition were in the range from 1.5 to $2.0 \times 10^{19} \mathrm{~m}^{-3}$. H-phases of up to $600 \mathrm{msec}$ were produced, and the discharges tended to be ELM-free if the plasmas became diverted at the start of the current flattop phase but ELMy if the plasma became diverted during current ramp-up. ELM characteristics depend also on gas fueling rate and divertor configuration/triangularity. Comprehensive descriptions of the NSTX Hmodes can be found in Maingi et al ${ }^{19,20}$ and Bush et al. ${ }^{17}$ Only NBI-heated discharges will be used for the study presented here, although H-modes with rf heating only have been observed. There are not, however, a sufficient number of rf-only H-modes to do any mean- 
ingful comparisons between threshold conditions in those and NB-heated discharges. In addition, no ohmic H-modes were observed.

What is found in the theory-experiment comparison in this work is that L-and H-mode NSTX plasmas are distinguishable within the frameworks of most of the theories, and this distinction is due primarily to differences in $\beta_{t}$ and $L_{n}$, the density gradient scale length, between the two states. The theories, however, appear to have little or no predictive capability. This is not necessarily surprising in light of the relatively limited set of parameters considered by the various theories and the possible importance of other effects. One such effect, evidence for which will be presented, is fast ion loss. Because of the low toroidal magnetic field of NSTX, the fast ion Larmor radius can be a signficant fraction of the minor radius $(1 / 5$ to $1 / 3)$ unlike that at conventional aspect ratio, and fast ion loss due to classical or MHD-related effects can be large. It will be shown how this loss can influence the L- to H- transition.

An example of an H-mode discharge is shown in Fig. 1. Close to $4 \mathrm{MW}$ of neutral beam power was injected into this $0.8 \mathrm{MA}$ discharge (top panel), and the $\mathrm{L}-\mathrm{H}$ transition as seen in the $D_{\alpha}$ and line-averaged density traces (middle panel) occurred at approximately $0.24 \mathrm{sec}$, shortly after the second beam source turned on. At the time of the transition, the time rate of change of the line-averaged density increased, and the stored energy (bottom panel), which was increasing due to the turning on of the second beam source, continued to increase. The stored energy reached a steady-state for over $0.3 \mathrm{sec}$, with the confinement enhancement factor, relative to the ITER98pby, 2 scaling, ${ }^{21}$ reaching 1.4 to 1.6. The confinement enhancement is calculated by normalizing the global confinement time (including the fast ion component) by the value given by the scaling expression, which is for the thermal confinement time. Calculations of fast ion energy content and losses are underway in order to determine the thermal confinement times, and, therefore, a more accurate thermal confinement enhancement factor.

The critical data used for this study come from the multi-point Thomson scattering 
(MPTS) diagnostic, which measured instantaneous electron temperature and density profiles every $16 \mathrm{msec}$. The MPTS profiles consisted of 20 spatial points, with the channels separated by approximately two to three $\mathrm{cm}$ in the outer portion of the plasma. Temperature, density, and gradients of these quantities were taken from spline fits to the MPTS data for this study. The spline fits used consisted of 100 radial points, with a radial grid of 1.3 $\mathrm{cm}$. Data were chosen at locations midway down the edge density gradient, similar to the location chosen for the DIII-D studies. ${ }^{9}$

Figures 2 and 3 show sets of MPTS profiles, both full and in the outer portion of the plasma ( $\mathrm{R} \geq 1.2 \mathrm{~m}$ ), for two discharges, one $0.7 \mathrm{msec}$ before the $\mathrm{L}-\mathrm{H}$ transition (Fig. 2), and one in the midst of the H-phase, $t_{L H}=0.252 \mathrm{sec}$ (Fig. 3). Also shown in each figure are the positions of the separatrix, as determined from EFIT magnetic reconstructions, ${ }^{18}$ and the "mid-gradient" location at which the relevant plasma parameters are measured or calculated. The mid-gradient location is determined from the positions of the knee and the separatrix. In these examples and for the points to be shown, the normalized poloidal flux at the mid-gradient location varied from 0.935 to 0.995 , but with most of the values being approximately 0.98 . The red triangles in the figures represent the actual data points whose statistical (photon count) errors are actually smaller than the triangle height. The blue lines are the spline fits to the data. What is clearly seen in the Fig. 3 is the development of an "ear" in the density profile during the H-phase, and the much larger edge density and density gradient than during the L-phase. During the latter portions of the H-phase, the central density fills in, and the density profile becomes flat. In these cases, the density gradient, as determined from the spline fit, is about a factor of two to three larger during the H-phase than just prior to the transition.

\section{B. Comparisons with Transition Theories}

For the comparison with theory, 225 different time points from 214 different discharges were used. The 225 time points were grouped by the following discharge phases and con- 
ditions: L-mode - 41; Dithering transitions - 6; times just prior to the L-H transition 79; ELM-free H-mode - 22; ELMy H-mode - 77 (64 with small ELMs and 13 with giant ELMs). For the L-H transition grouping, only times where the MPTS measurement was within $8 \mathrm{msec}$ prior to the transition were used. For this grouping, the range of $\Delta t_{L H}=t_{L H}-t_{M P T S}$ was 0.0 to $7.9 \mathrm{msec}$, with an average value of $4.1 \mathrm{msec}$ and a standard deviation of 2.4 msec. In the H-mode grouping, for both ELM-free and ELMy points, the time of interest was taken within the well-developed H-mode phase, with the average $t_{M P T S}-t_{L H}=143.7 \pm 74.4 \mathrm{msec}$. Dividing the data into these groupings is important for both determining any significant differences among the various phases as well as, particularly with the L-H grouping, determining whether there are any theory-based parameters that can be used as predictors for impending transitons.

The results of the comparisons are shown in Fig. 4. Data is plotted in terms of the relevant theoretical quantities for the drift-resistive ballooning mode (Fig. 4a), the peeling mode (Fig. 4b), and the drift-Alfven mode (Fig. 4c). The ELMy and ELM-free data are grouped together in the H-mode group; the ELMy discharges generally lie at the upper range of the $\mathrm{H}$-mode groupings for the three different cases. The dithering grouping is indistinct from the L-H group of points (i.e., times taken just before the transition) in the figure, and, therefore, have not been separated from the L-H group.

In the drift-resistive ballooning mode theory, the two controlling parameters are the MHD ballooning parameter, $\alpha_{M H D}=-R q^{2} d \beta_{t} / d r$ where $R$ is radius, $q$ is the inverse rotational transform and $\beta_{t}$ is as defined previously, and the diamagnetic parameter, $\alpha_{D I A}$, which reduces to $\left(\rho_{s} \Omega_{e}\right)^{1 / 2} /\left[2^{5 / 4} \pi\left(R L_{n}\right)^{1 / 4}(1+\tau) q \nu_{e i}^{1 / 2}\right]$ where $\rho_{s}=c_{s} / \Omega_{c i}, \tau=T_{i} / T_{e}$, and $L_{n}$ is the density scale length, with all quantities taken locally and $\tau$ taken to be one. Here, $\Omega_{e}$ is electron gyrofrequency, $\nu_{e i}$ is electron-ion collision frequency, $c_{s}$ is the sound speed and $\Omega_{c i}$ is the ion gyrofrequency. The above expressions are valid for a shifted circle geometry, which is not entirely appropriate for NSTX; however, the difference in results from the above expressions and those with general geometry and shaping corrections is 
small. ${ }^{22}$ Also, for these comparisons, only the electron profiles were used to determine $L_{n}, L_{p}, d \beta_{t} / d r$, etc.

In the drift-resistive ballooning theory, higher $\alpha_{D I A}$ causes enhanced poloidal flow shear which serves to suppress turbulence. In addition, increased $\alpha_{M H D}$ also leads to transport suppression so that a reduced transport (H-mode) region forms at large values of $\alpha_{D I A}$ and $\alpha_{M H D}$. The NSTX results shown in Fig. 4a show a clear separation between the points in the H-phase and those points either remaining in the L-mode or in the transition groupings, indicative of the higher local $\beta$ and $\beta^{\prime}$ during the H-phase. What is striking in the plot is that this separation occurs at a value of $\alpha_{M H D} \simeq 1$ to 2 over almost an order of magnitude variation in $\alpha_{D I A}$, consistent with expectations from a bifurcation mechanism for which $\alpha_{M H D} \simeq 1$ to 2 is the threshold. In contrast, the approximate boundary for the H-mode, as given by the theory, is denoted by the shaded region in Fig. 4a. As can be seen, there is not quantitative agreement between the theory and the experimentally observed boundaries, with the experimental threshold for $\alpha_{D I A}$ being about a factor of two lower than the theoretical threshold $(\simeq 0.6)$, and the experimental threshold for $\alpha_{M H D}$ being a factor of two to three higher than that of theory $(\simeq 0.4)$.

Of particular significance also is that little difference is seen among the L-mode and L-H transition groupings, although it appears from the plot (and also in Fig. 4b) that the maximum $\alpha_{M H D}$ values of the L-H grouping lie slightly above those of the group of Lmode points. This systematic difference, however, is within the error bars, the typical size of which is shown on each plot. In addition, no systematic dependence between the magnitude of $\alpha_{M H D}$ and temporal proximity to the $\mathrm{L}-\mathrm{H}$ transition was observed; that is, $\alpha_{M H D}$ was not systematically higher for $\mathrm{L}-\mathrm{H}$ points for times that were closest to the time of the L-H transition. These results indicate that changes in the relevant profile characteristics on time scales just prior to the transition are subtle. The error bars were estimated from the appropriate sum of the relative errors of the individual parameters that make up these variables. The largest source of quantifiable random error comes from the spline fits to 
the Thomson scattering data, and their derivatives. Consequently, even with the size of the error bars, the above conclusion regarding the threshold value of $\alpha_{M H D}$ is still valid. However, the changes leading up to the transition are unresolvable within experimental error, limiting the usefulness of this parameter as a dynamic predictor of an impending transition.

In the peeling mode formulation, the key parameters are the electron collisionality, $\nu_{e} \propto n_{e} \ln \Lambda Z_{e f f} q R_{0}^{5 / 2} /\left(T_{e}^{2} r^{3 / 2}\right)$ and, once again $\alpha_{M H D}$. The peeling mode is stabilized by edge pressure gradient, but destabilized by edge current. Therefore, high collisionality is needed to reduce the bootstrap current driven by large $\nabla p$, thus forming a space of reduced transport at large $\alpha_{M H D}$ and $\nu_{e}$. Fig. $4 \mathrm{~b}$ also shows a clear separation between the H-phase points and the L-mode or transition groupings, with the $\alpha_{M H D} \simeq 1$ to 2 threshold extending over an order of magnitude in $\nu_{e}^{\star}$, and with the L and L-H groupings overlapping over this range as well. As for the drift-resistive ballooning theory, there is some discrepancy between the experimental and theoretical thresholds (the H-mode region as defined by theory is again shown by the shaded region). The theoretical threshold for $\nu_{e}^{\star}$ is $\simeq 1$, while experimentally H-mode values down to 0.2 are observed, with $\mathrm{L}$ and $\mathrm{L}-\mathrm{H}$ points above the theoretical threshold as well.

The same results are also seen in Fig. $4 \mathrm{c}$, in which $\beta_{n} /\left(1+\nu_{n}^{2 / 3}\right)=\left(\frac{M_{i}}{m_{e}}\right)^{1 / 2} \alpha_{M H D} /(s q(1+$ $\left.\left.\nu_{n}^{2 / 3}\right)\right)$ where $s=r q^{\prime} / q$, and $\nu_{n}=\nu_{e}^{\star}\left(M_{i} L_{p} / s m_{e} q R\right)^{1 / 2}$, is plotted against $\nu_{n}$. In this driftAlfven mode theory, transport is suppressed above the threshold $\beta_{n}>1+\nu_{n}^{2 / 3}$. While the scatter is much greater for this theory than for the others, the NSTX data do show that the $\mathrm{H}$-phase points generally lie above a minimum $\beta_{n} /\left(1+\nu_{n}^{2 / 3}\right)$ value, consistent with the COMPASS-D and DIII-D results, which for NSTX is $\simeq 9$ (the theoretical threshold is 1 ). Again, the transition grouping appears to overlap with the L-phase grouping, and both can lie above this "threshold" as well.

Comparisons with the above theories were also made at locations close to the knee, where the normalized poloidal flux varied from 0.91 to 0.98 , with most of the values being approximately 0.955 . The results of the comparisons at this location were similar to those 
at the mid-gradient location.

The NSTX edge data also do not indicate any obvious critical temperature for the L-H transition, as there is no simple separation of measured edge $T_{e}$ values for the L-mode, L-H or H-mode groups of points from shot-to-shot. The existence or not of a critical edge temperature can be investigated further within the framework of the zonal flow turbulence suppression model, ${ }^{7}$ in which the critical $T_{e}$ is given by $L_{n}^{1 / 2} B_{T}^{2 / 3}\left(Z_{e f f}^{2} / R\right)^{1 / 6}$. In Fig. 5 is shown a time evolution of $\frac{T_{e}}{T_{e, c r i t}}$ (top panel) and $T_{e}$ (bottom panel) at a position $5 \mathrm{~cm}$ inside the separatrix for a discharge that underwent an $\mathrm{L}-\mathrm{H}$ transition at a time indicated by the vertical line. A similar plot is shown in Fig. 6 for a lower power, higher current discharge that remained in the L-mode. As can be seen in Fig. 5, the $\frac{T_{e}}{T_{e, c r i t}}$ reached a value of approximately 0.3 (corresponding to $T_{e}=50 \mathrm{eV}$ ), and then remained at that level for about 40 msec until it underwent a transition to the H-mode. In some cases, the $\frac{T_{e}}{T_{e, c r i t}}$ was seen to increase monotonically to the point at which it transitioned, while in others, such as the case shown here, the "threshold" value was reached and the discharge remained there for some time before undergoing a transition. In other cases yet, the value of $\frac{T_{e}}{T_{e, c r i t}}$ at which the discharge transitioned was actually lower than the values of this parameter at preceding times. This alone suggests that $\frac{T_{e}}{T_{e, c r i t}}$ is not sufficient to determine the L-H transition for NSTX plasmas. It is seen in Fig. 6, where the discharge remained in the L-mode, that $\frac{T_{e}}{T_{e, c r i t}}$ achieved values greater than the value at the transition in Fig. 5 (up to 0.6), and remained at that higher level. Despite this, and the fact that $T_{e} \geq 50 \mathrm{eV}$ during this period of time, no transition was observed. In general, once a transition occurred, $\frac{T_{e}}{T_{e, c r i t}}$ increased as the large density gradient developed.

The result discussed above can also be studied statistically. In Fig. 7 are plotted histograms of the measured edge $T_{e}$ normalized to the critical $T_{e}$ for time slices just before the L-H transition (top panel) and for time slices in discharges that remained in L-mode (bottom panel). The values shown are taken $5 \mathrm{~cm}$ inside the separatrix $\left(\psi_{n}=0.955\right.$ to 0.975 , where $\psi_{n}$ is normalized poloidal flux), but similar results are found for radial positions 
anywhere from $3\left(\psi_{n}=0.975\right.$ to 0.995$)$ to $6\left(\psi_{n}=0.950\right.$ to 0.97$) \mathrm{cm}$ inside as well. The histograms show a wide range of $\frac{T_{e}}{T_{e, c r i t}}$ for the $\mathrm{L}-\mathrm{H}$ points; there is no minimum value as was seen for DIII-D. ${ }^{7}$ The L-H distribution covers a range similar to that covered by the L-mode distribution of time slices, indicating no separation between these groups of points (i.e., the L-H distribution is not skewed to higher values of $\frac{T_{e}}{T_{e, c r i t}}$, as might be expected if this were a controlling parameter).

The results from Figs. 5 to 7 indicate that if there is a local $T_{e}$ threshold for an L-H transition in NSTX, it has a more complicated scaling than that given by Guzdar et al.. ${ }^{7} \mathrm{~A}$ more detailed experimental study of whether or not a $T_{e}$ threshold exists is planned, and this will involve controlling the edge $T_{e}$ through gas or impurity puffing for otherwise constant discharge conditions.

\section{Influence of Fast Ions}

The comparisons presented thus far indicate that the theories as such cannot explain the L-H transition dynamics for NSTX. The theories may need further development to enhance their validity in low aspect ratio regimes, but they also, however, may not take into account effects that can influence the L-H transition. One such effect is the non-ambipolar loss of beam ions, which can influence the edge radial electric field and its shear, and thus the development of an edge transport barrier. Once piece of evidence that this loss can aid the L-H transition in NSTX. is the current dependence of the L-H threshold power as reported in Bush et al. ${ }^{17}$ When the total power required for an $\mathrm{L}-\mathrm{H}$ transition is normalized according to the threshold scaling in Eq. 1 of Snipes et al., ${ }^{23}$ to take into account the conventional density, toroidal field and plasma size and shape dependences, a residual dependence of this normalized threshold power on plasma current is found. The threshold power triples going from 0.6 to 1.0 MA. For 0.6 MA, $P_{l o s s}=0.53 \mathrm{MW}$ was required for an L-H transition $\left(P_{\text {loss }} / P_{\text {scaling }} \simeq 2.5\right)$, while for $1.0 \mathrm{MA}, P_{\text {loss }}=1.56 \mathrm{MW}\left(P_{\text {loss }} / P_{\text {scaling }} \simeq 7\right)$. A key difference between the two current levels which may lead to the current dependence for 
the reasons just mentioned is the expected beam ion loss through bad orbits and chargeexchange, which is $40 \%$ for $0.6 \mathrm{MA}$ but only $5 \%$ at $1 \mathrm{MA}$.

Transient fast ion loss apparently can also affect the L-H transition. An example of this is shown in Fig. 8, which is an expanded time period of a sawtooth-free discharge near the time of an L-H transition. Plotted in the figure is the perturbed magnetic field (top), neutron signal (middle) and $\mathrm{D}_{\alpha}$ (bottom). A transient drop in $\mathrm{D}_{\alpha}$ which could signify a transient entry into the H-mode (dither), and which was associated with a burst of MHD and a drop in the neutron flux, occured at 0.231 seconds. The discharge entered into a sustained H-phase at $0.244 \mathrm{sec}$, and this L-H transition was associated with a larger MHD burst and larger drop in the neutron flux (10\%). The MHD bursts associated with these drops in neutron flux and $\mathrm{D}_{\alpha}$ are believed to be bounce precession fishbones. ${ }^{24}$ In both cases, the $\mathrm{D}_{\alpha}$ drops were delayed from the neutron flux drop by about 1 to 2 msec. The drops in neutron flux indicates a loss or redistribution of the fast ion population, as little or no change in density or temperature was associated with these events. The L-H transition was associated with the largest neutron drop, and thus the largest fast ion loss (or redistribution). The drop in the neutron rate corresponds to a similar reduction in the fast ion reactions creating these neutrons since the neutrons are produced primarily by beam-target reactions. That magnitude loss corresponds to over $10^{17} \mathrm{~m}^{-3}$ fast ions, which, according to Parail et al., ${ }^{25}$ corresponds to an incremental radial electric field of approximately $3 \mathrm{kV} / \mathrm{m}$. While this $E_{r}$ is modest compared to values at conventional aspect ratio, ${ }^{26}$ the associated rotation and possibly rotational shear can be large owing to the order of magnitude lower $B_{T}$ on NSTX than that at conventional aspect ratio. It is believed that it is the radial electric field and shear created by the loss of fast ions that influence the L-H transition, as there is no heat pulse associated with these MHD bursts that could trigger the transition. Other discharges reveal series of $\mathrm{D}_{\alpha}$ and neutron drops, representing an extended dithering period, where the ultimate L-H transition here also is associated with the largest neutron drop, and, therefore, largest fast ion loss. 


\section{SUMMARY}

In summary, this paper presents the first attempt to relate edge measurements in a high power Spherical Torus to transport suppression theories in order to understand mechanisms that may lead to the transition from the L- to the H-mode. Overall, the results challenge present theory and suggest paths for further development of these frameworks. Data measured by the MPTS diagnostic were broken into different groups, and for the theories studied, clear differences between the H-phase points and L-mode and transition groups are seen. These differences can be attributed primarily to the larger edge pressure and density gradients and density during the H-phase, and the strong dependence of the respective theory variables on these parameters. In particular, the separation of these data appear to be consistent with a bifurcation mechanism with $\alpha_{M H D} \simeq 1$ to 2 as a threshold. The experimental boundaries separating the groups are not in strict quantitative agreement with those expected from theory, and the reason for this may be due, in part, to the fact that only the electron profile data were used to compute the local $\beta_{t}$ and gradients. Little distinction is seen between the L-H group and the L-mode group within the error bars, indicating that the changes in the parameters, most notably $\alpha_{M H D}$, leading up to the transition are subtle, and any differences in discharges that remain in the L-mode and those in the L-mode that ultimately transition are not readily resolvable within experimental error either statistically or dynamically. In addition, the NSTX data show no simple temperature threshold for transitioning into the H-mode. These results indicate that the theories, at present, have limited predictive capability to within the temporal and spatial resolution of the dataset used, and for the low aspect ratio geometry and discharge characteristics.

Key effects may also be missing from the theories. One of may be the possible influence of fast ion loss, which can be significant for STs, and the effect of turbulence suppression due to radial electric fields and field shear on the $\mathrm{L}-\mathrm{H}$ transition. A model of non-ambipolar losses leading to L-H transitions was developed by Itoh and Itoh. ${ }^{28}$ In 
this model, non-ambipolar electron losses were induced by microturbulence, while nonambipolar ion losses were due to ion-ion collisions, and, therefore, their derived condition for bifurcation depended strongly on collisionality. It is noteworthy that the NSTX dataset overall did not show a dependence of the threshold on collisionality (Figs. 4b and 4c); however, more work is planned which will attempt to identify key parameters and dependences associated with the fast ion loss that can lead to the L-H transitions in NSTX. Furthermore, estimates of fast ion loss and generated radial electric fields will be made using the expected mode structures as computed by MHD stability codes coupled to calculations of the distribution of fast ions.

\section{ACKNOWLEDGEMENTS}

We thank Tom Carlstrom, Parvez Guzdar and Jim Drake for discussions about this work and the NSTX Physics and Operations teams for their support. This work was supported by US Department of Energy Contract DE-AC02-76CH03073 at the Princeton Plasma Physics Laboratory, DE-AC05-00OR22725 at Oak Ridge National Laboratory, 


\section{References}

${ }^{1}$ F. Wagner and G. Becker and K. Behringer and et al., Phys. Rev. Lett. 49, 1408 (1982).

${ }^{2}$ J.D. Strachan and M. Bitter and A.T. Ramsey and et al., Phys. Rev. Lett. 58, 1004 (1987).

${ }^{3}$ B.N. Rogers and J.F. Drake and A. Zeiler, Phys. Rev. Lett. 81, 4396 (1998).

${ }^{4}$ P.N. Guzdar and R.G. Kleva and A. Das and P.K. Kaw, Phys. Rev. Lett. 87, 015001 (2001).

${ }^{5}$ H. Wilson and J.W. Connor and A.R. Field and S.J. Fielding and R.L. Miller and L.L. Lao and J.R. Ferron and A.D. Turnbull, Phys. Plasmas 6, 1925 (1999).

${ }^{6}$ O. Pogutse and Y. Igitkhanov and W. Kerner and G. Janeschitz and J.G Cordey, The alfven drift-wave instability and the scaling of the edge temperature at the L-H transition, in Controlled Fusion and Plasma Physics, volume 21A, page 1545, European Physical Society, Geneva, (Proc. 24th Eur. Conf. Berchtesgaden, 1997), 1997.

${ }^{7}$ P. Guzdar and R. Kleva and R. Groebner and P. Gohil, Phys. Rev. Lett. 89, 265004 (2002).

${ }^{8}$ A. Hubbard and R.L. Boivin and J.F. Drake and M. Greenwald and Y. In and J.H. Irby and B.N. Rogers and J.A. Snipes, Local variables affecting H-mode threshold on Alcator C-Mod, in Plasma Physics and Controlled Fusion, volume 40, page 689, Institute of Physics, (Proc. 6th IAEA Tech. Comm. Mtg on H-mode Physics, 1997), 1998.

${ }^{9}$ T. Carlstrom and K.H. Burrell and R.J. Groebner and et al., Nucl. Fusion 39, 1941 (1999).

${ }^{10}$ P. Gohil, L.R. Baylor, T.C. Jernigan, K.H. Burrell, and T.N. Carlstrom, Phys. Rev. Lett. 86, 644 (2001).

${ }^{11}$ S.J. Fielding and P.G. Carolan and J.W. Connor and et al., Nucl. Fusion 41, 909 (2001). 
${ }^{12}$ W. Suttrop and M. Kaufmann and H.J. deBlank and et al., Plasma Physics and Controlled Fusion 39, 2051 (1997).

${ }^{13}$ E. Righi and D. Bartlett and G.D. Conway and et al., The role of edge parameters in the H-mode transition on JET, in Controlled Fusion and Plasma Physics, volume 21A, page 93, European Physical Society, Geneva, (Proc. 24th Eur. Conf. Berchtesgaden, 1997), 1997.

${ }^{14}$ T. Fukuda and M. Sato and T. Takizuka and et al., Hh-mode transition and power threshold in JT-60U, in Fusion Energy, volume 1, page 857, IAEA, Vienna, (Proc. 16th IAEA Fusion Energy Conference, Montreal, Canalda, 1996), 1996.

${ }^{15}$ S. Kaye and M. Ono and Y.K.M.Peng and et al., Fusion Technology 36, 16 (1999).

${ }^{16}$ S. Kaye and the ITER Confinement Database Working Group, Nucl. Fusion 37, 1303 (1997).

${ }^{17}$ C. Bush and and M.G. Bell and R.E. Bell and et al., Phys. of Plasmas 101755 (2003).

${ }^{18}$ S.A. Sabbagh and S.M. Kaye and J. Menard and et al., Nuc. Fusion 411601 (2001).

${ }^{19}$ R. Maingi and M.G. Bell and R.E. Bell and et al., Phys. Rev. Lett. 88, 035003 (2002).

${ }^{20}$ R. Maingi and M.G. Bell and R.E. Bell and et al., H-modes research in NSTX, 19th IAEA Fusion Energy Conference, Lyon, France, 2002, accepted for publicaiton in Nuc. Fusion, 2003.

${ }^{21}$ The ITER Team, Nuc. Fusion 392175 (1999).

${ }^{22}$ T. Carlstrom, J. Drake, private communications, 2001.

${ }^{23}$ J. Snipes and the ITER Confinement and H-mode Threshold Database Working Group, Multi-machine global confinement and H-mode threshold analysis, 19th IAEA Fusion Energy Conference, Lyon, France, 2002. 
${ }^{24}$ E. Fredrickson and et al., Wave driven fast ion loss in the National Spherical Torus Experiment, accepted for publication in Phys. of Plasmas, 2003.

${ }^{25}$ V.V. Parail and A.Y. Guo and J. Lingertat, Nuc. Fusion 39, 369 (1999).

${ }^{26}$ R.J. Groebner, Phys. Fluids B 7, 2343 (1993).

${ }^{27}$ R.J. Akers and G.F. Counsell and A. Sykes and et al., Phys. Rev. Lett. 88, 035002 (2002).

${ }^{28}$ S.-I. Itoh and K. Itoh, Phys. Rev. Lett. 60, 2276 (1988). 


\section{Figure Captions}

Figure 1 NSTX H-mode discharge evolution.

Figure 2 MPTS electron temperature and density profiles measured just before an L-H transition and The red triangles are data points, the blue curves are spline fits to the data.

Figure 3 MPTS electron temperature and density profiles measured during a well developed H-phase. The red triangles are data points, the blue curves are spline fits to the data.

Figure 4 Comparisons between experimental data and theory for (a) drift-resistive ballooning modes, (b) peeling modes and (c) drift-Alfven modes. The shaded region in each plot indicate the theoretical prediction of H-mode access.

Figure 5 Time evolution of $T_{e}$ normalized to the critical temperature given by Guzdar et $\mathrm{al}^{7}$ (top panel) and $T_{e}$ (bottom panel) for a discharge that transitioned into the $\mathrm{H}-$ mode at a time denoted by the vertical line The solid triangles are the actual times at which the data were taken. The discharge parameters were $I_{p}=0.8 \mathrm{MA}, B_{T}=0.5 \mathrm{~T}$, $P_{i n j}=4.1 \mathrm{MW}$, and $\bar{n}_{e}=2.5 \times 10^{19} \mathrm{~m}^{-3}$ at the time of the transition.

Figure 6 Time evolution of $T_{e}$ normalized to the critical temperature given by Guzdar et $\mathrm{al}^{7}$ (top panel) and $T_{e}$ (bottom panel) for a discharge that remained in the L-mode. The solid triangles are the actual times at which the data were taken. The discharge parameters were $I_{p}=1.0 \mathrm{MA}, B_{T}=0.44 \mathrm{~T}, P_{i n j}=1.6 \mathrm{MW}$, and $\bar{n}_{e}=2.1 \times 10^{19} \mathrm{~m}^{-3}$ at $0.25 \mathrm{sec}$.

Figure 7 Histograms of $T_{e}$ normalized to the critical temperature given by Guzdar et $\mathrm{al}^{7} 5$ $\mathrm{cm}$ inside the separatrix for time points just prior to the $\mathrm{L}-\mathrm{H}$ transition (top) and in discharges that remained in the L-phase (bottom). 
Figure 8 Expanded time evolution of a discharge showing a dither $(t=0.231 \mathrm{sec})$ prior to the $\mathrm{L}-\mathrm{H}$ transition $(\mathrm{t}=0.242 \mathrm{sec})$. Plotted is the magnetic field fluctuation (top), neutron flux (middle) and $\mathrm{D}_{\alpha}$ signals (bottom). 


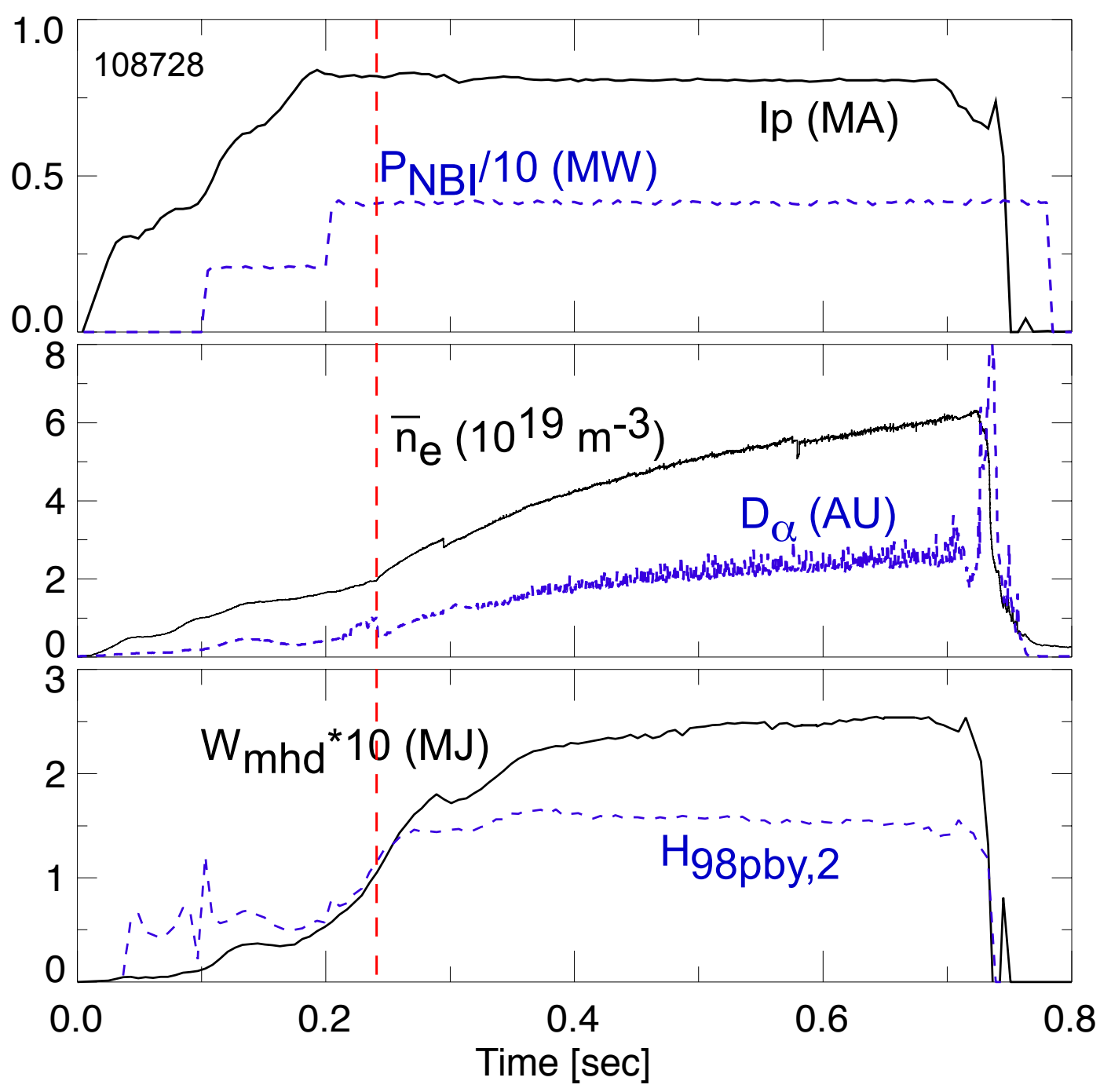

Figure 1: 

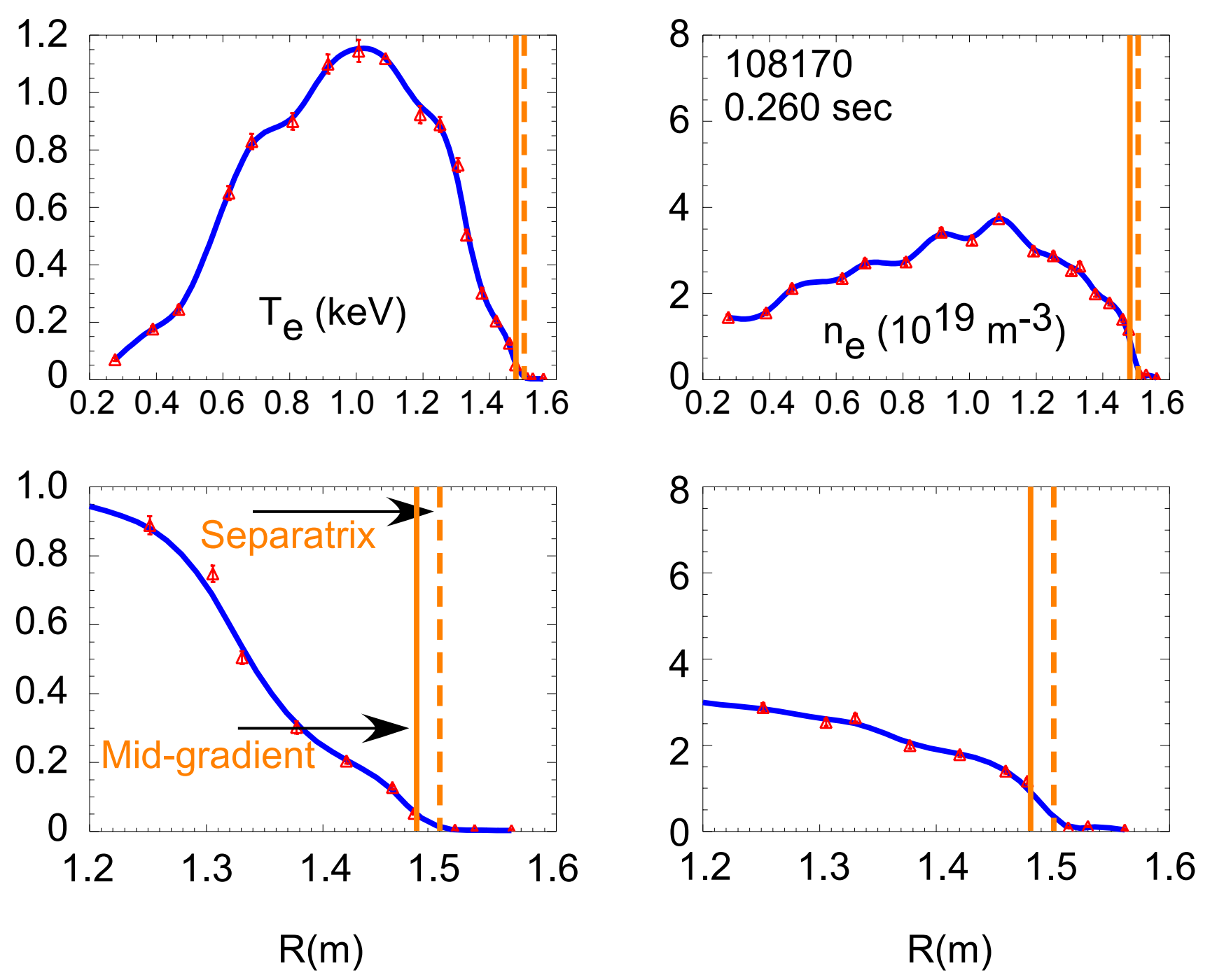

Figure 2: 

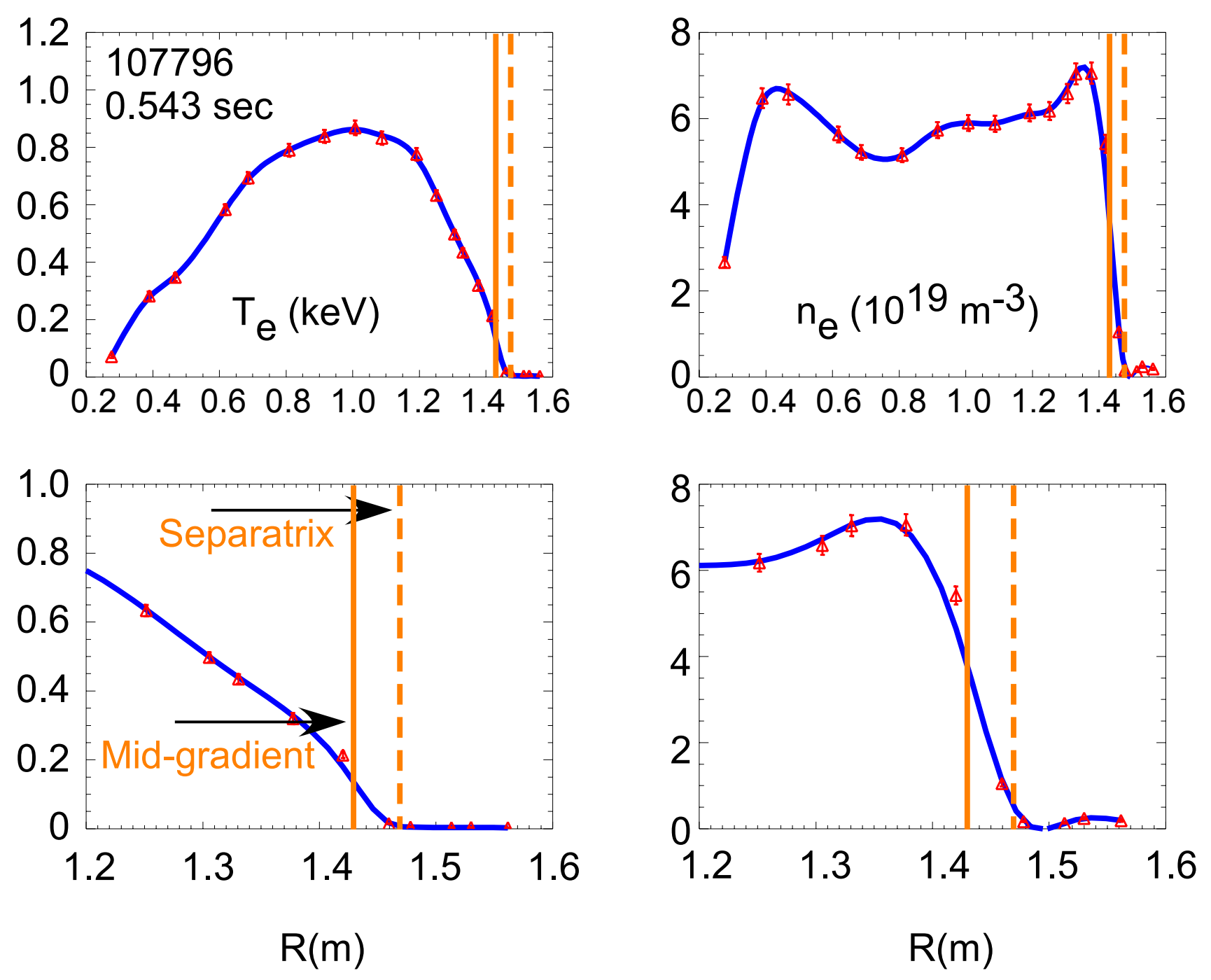

Figure 3: 
- L-mode - L-H $\Delta$ H-mode

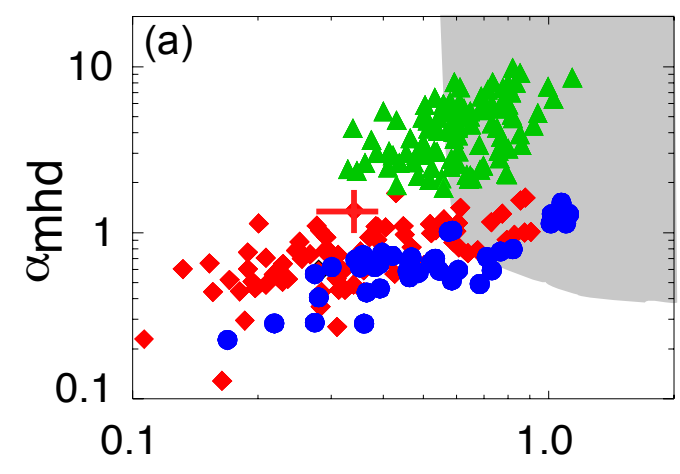

$\alpha_{\text {dia }}$
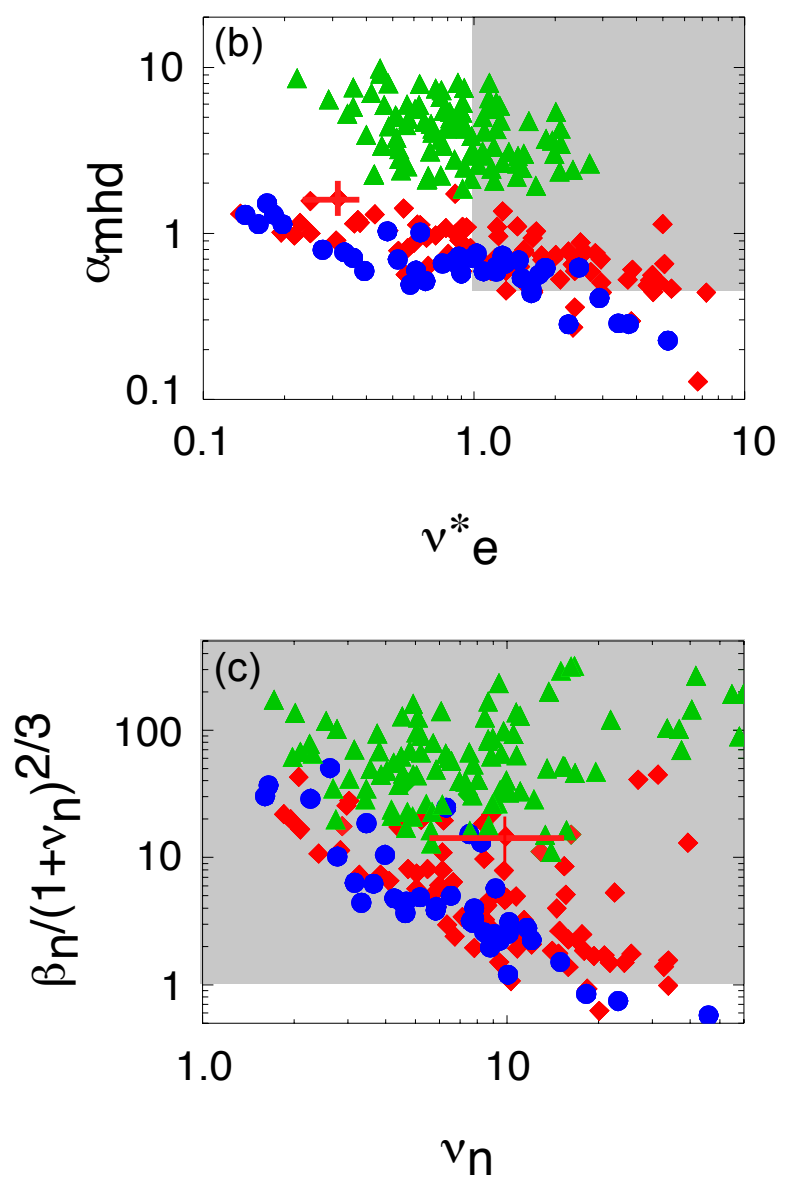

Figure 4: 

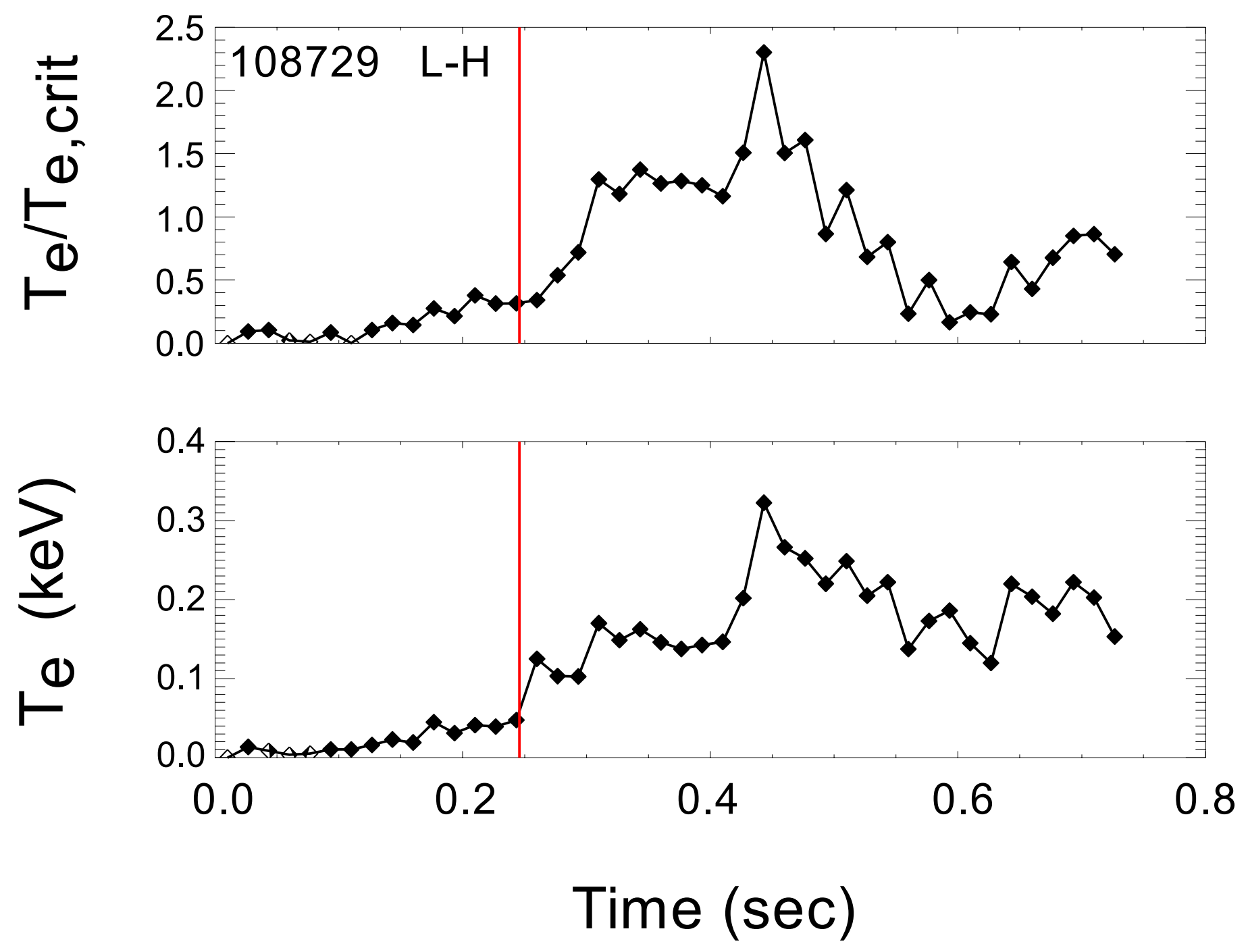

Figure 5: 

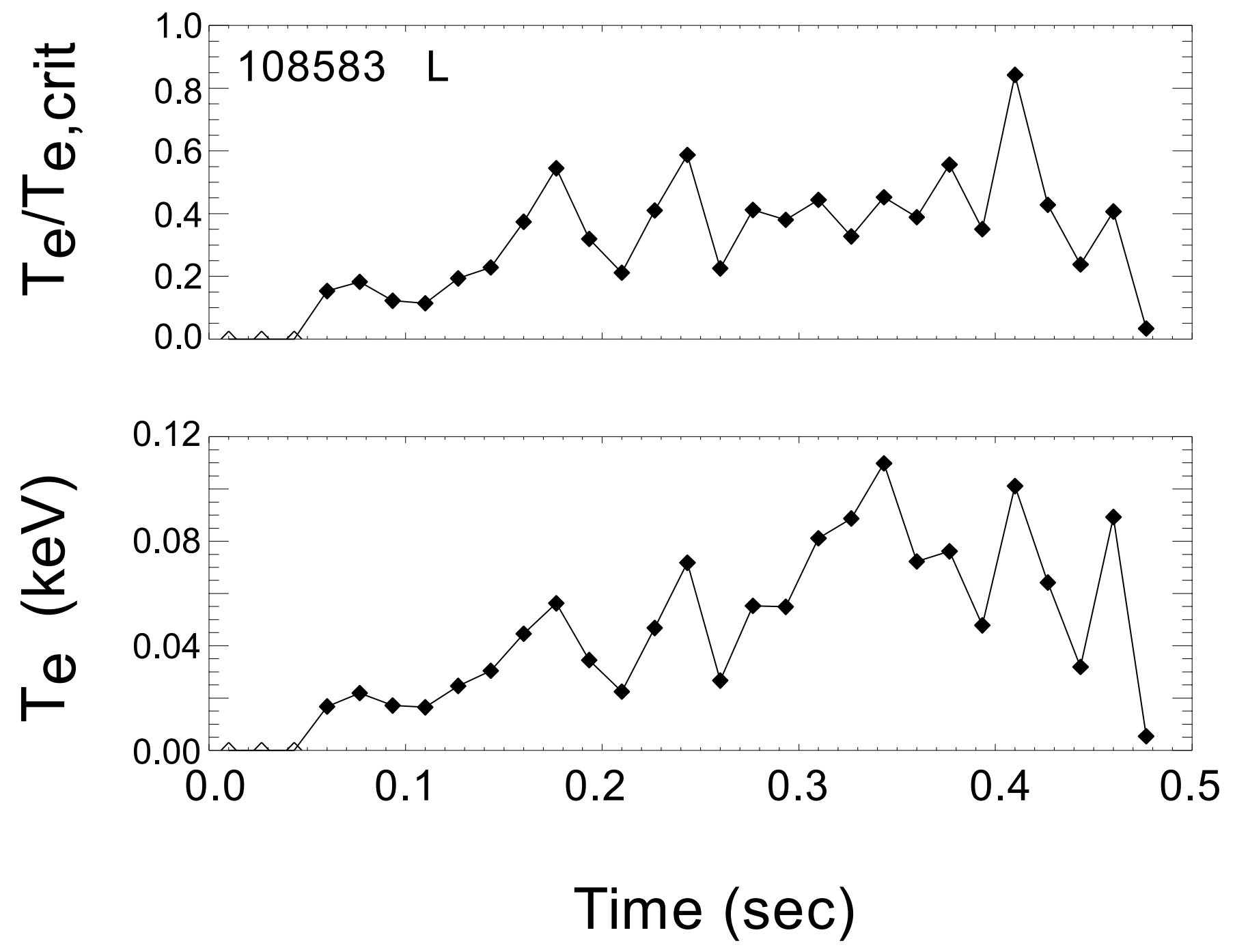

Figure 6: 

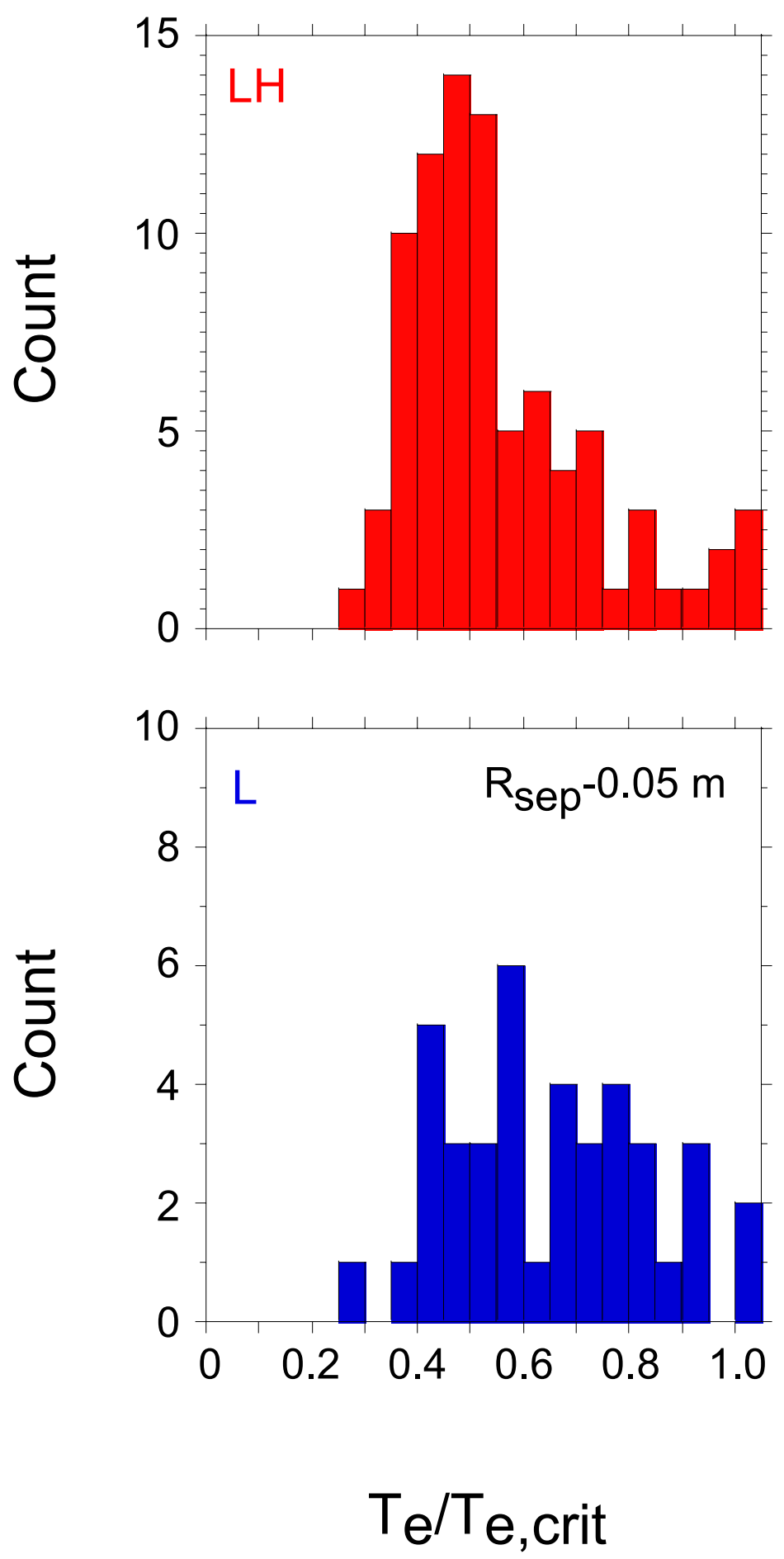

Figure 7: 


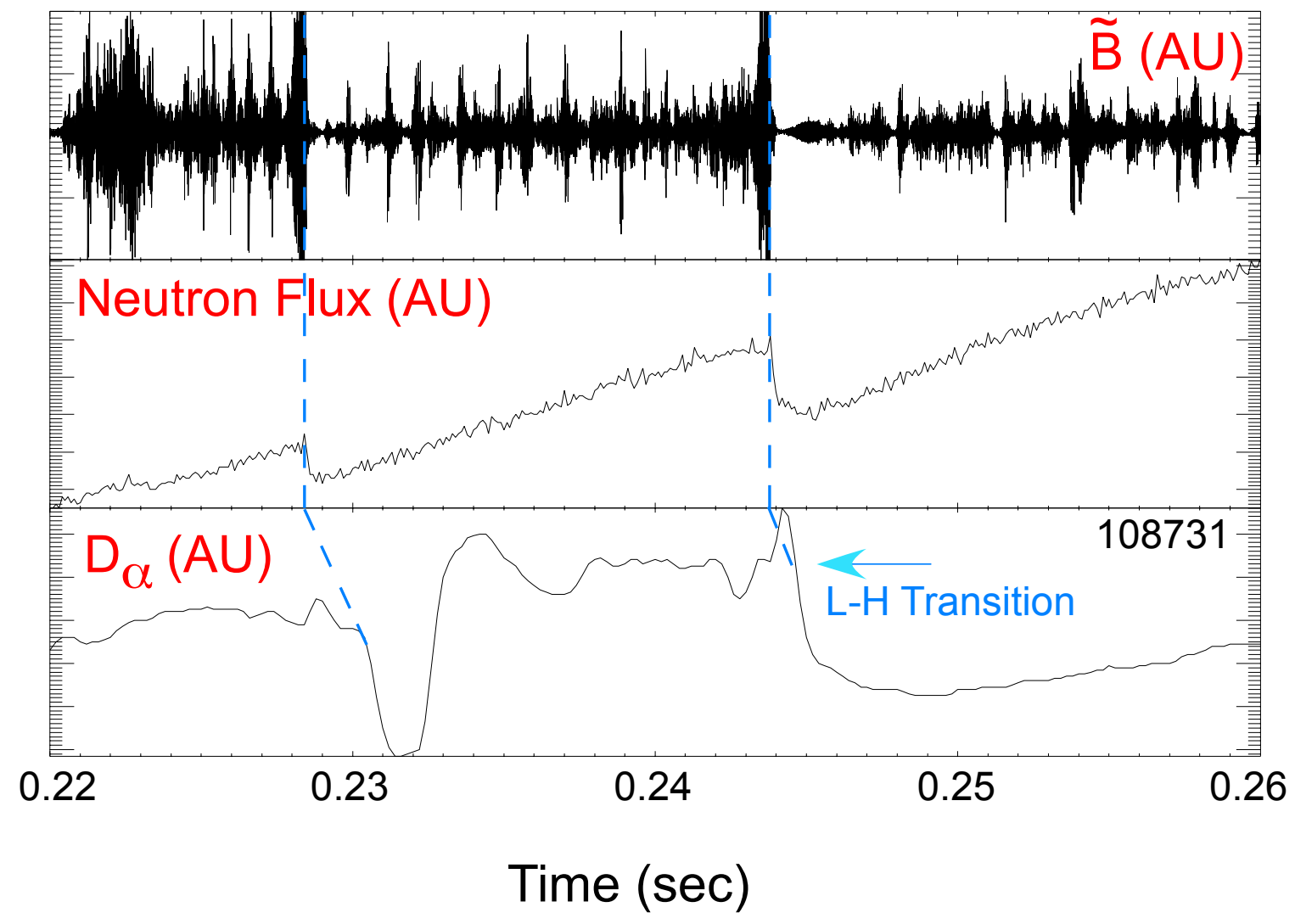

Figure 8: 


\section{External Distribution}

Plasma Research Laboratory, Australian National University, Australia

Professor I.R. Jones, Flinders University, Australia

Professor João Canalle, Instituto de Fisica DEQ/IF - UERJ, Brazil

Mr. Gerson O. Ludwig, Instituto Nacional de Pesquisas, Brazil

Dr. P.H. Sakanaka, Instituto Fisica, Brazil

The Librarian, Culham Laboratory, England

Mrs. S.A. Hutchinson, JET Library, England

Professor M.N. Bussac, Ecole Polytechnique, France

Librarian, Max-Planck-Institut für Plasmaphysik, Germany

Jolan Moldvai, Reports Library, Hungarian Academy of Sciences, Central Research Institute for Physics, Hungary

Dr. P. Kaw, Institute for Plasma Research, India

Ms. P.J. Pathak, Librarian, Institute for Plasma Research, India

Ms. Clelia De Palo, Associazione EURATOM-ENEA, Italy

Dr. G. Grosso, Instituto di Fisica del Plasma, Italy

Librarian, Naka Fusion Research Establishment, JAERI, Japan

Library, Laboratory for Complex Energy Processes, Institute for Advanced Study, Kyoto University, Japan

Research Information Center, National Institute for Fusion Science, Japan

Dr. O. Mitarai, Kyushu Tokai University, Japan

Dr. Jiangang Li, Institute of Plasma Physics, Chinese Academy of Sciences, People's Republic of China

Professor Yuping Huo, School of Physical Science and Technology, People's Republic of China

Library, Academia Sinica, Institute of Plasma Physics, People's Republic of China

Librarian, Institute of Physics, Chinese Academy of Sciences, People's Republic of China

Dr. S. Mirnov, TRINITI, Troitsk, Russian Federation, Russia

Dr. V.S. Strelkov, Kurchatov Institute, Russian Federation, Russia

Professor Peter Lukac, Katedra Fyziky Plazmy MFF UK, Mlynska dolina F-2, Komenskeho Univerzita, SK-842 15 Bratislava, Slovakia

Dr. G.S. Lee, Korea Basic Science Institute, South Korea

Institute for Plasma Research, University of Maryland, USA

Librarian, Fusion Energy Division, Oak Ridge National Laboratory, USA

Librarian, Institute of Fusion Studies, University of Texas, USA

Librarian, Magnetic Fusion Program, Lawrence Livermore National Laboratory, USA

Library, General Atomics, USA

Plasma Physics Group, Fusion Energy Research Program, University of California at San Diego, USA

Plasma Physics Library, Columbia University, USA

Alkesh Punjabi, Center for Fusion Research and Training, Hampton University, USA

Dr. W.M. Stacey, Fusion Research Center, Georgia Institute of Technology, USA

Dr. John Willis, U.S. Department of Energy, Office of Fusion Energy Sciences, USA

Mr. Paul H. Wright, Indianapolis, Indiana, USA 
The Princeton Plasma Physics Laboratory is operated by Princeton University under contract with the U.S. Department of Energy.

\author{
Information Services \\ Princeton Plasma Physics Laboratory \\ P.O. Box 451 \\ Princeton, NJ 08543
}

Phone: 609-243-2750

Fax: 609-243-2751

e-mail: pppl_info@pppl.gov

Internet Address: http://www.pppl.gov 